\title{
Adaptive mesh and geodesically sliced Schwarzschild spacetime in 3+1 dimensions
}

\author{
Bernd Brügmann* \\ Max-Planck-Institut für Gravitationsphysik, Schlaatzweg 1, 14473 Potsdam, Germany
}

(Received 21 August 1996)

\begin{abstract}
We present the first results obtained with a $(3+1)$-dimensional adaptive mesh code in numerical general relativity. The adaptive mesh is used in conjunction with a standard ADM code for the evolution of a dynamically sliced Schwarzschild spacetime (geodesic slicing). We argue that the adaptive mesh is particularly natural in the context of general relativity, where apart from adaptive mesh refinement for numerical efficiency one may want to use the built in flexibility to do numerical relativity on coordinate patches.

[S0556-2821(96)02724-5]
\end{abstract}

PACS number(s): 04.25.Dm, 95.30.Sf, 97.60.Lf

\section{INTRODUCTION}

One of the stepping stones towards unrestricted $(3+1)$ dimensional numerical general relativity is the study of Schwarzschild spacetime. Spacetime singularities are one of the two characteristic features of vacuum general relativity, the other being gravitational waves. We certainly have to learn how to deal with a single, static black hole numerically if we want to treat astrophysically more interesting scenarios such as the collision of two black holes, the final stage of which is again a single static black hole.

Static, spherically symmetric Schwarzschild space-time turns into a rather challenging test case for standard $(3+1)$ dimensional numerical evolution schemes if one does not make use of the spherical symmetry other than in the initial data, if one uses Cartesian coordinates, and if one uses the freedom in the $3+1$ decomposition to define hypersurfaces on which the metric components evolve in time. This is what we implement here, following closely the work of Anninos et al. on 3+1 [1], which in turn is based on Bernstein et al. on $1+1$ [2] (by $n+1$ we denote the use of one time and $n$ space variables).

"Adaptive mesh" refers to a general technique for numerical evolution problems based on discrete grids, the basic idea being that one puts the points where one needs them for a given numerical accuracy. While traditionally the domain of numerical computation is taken to be a single, fixed rectangular grid (with several field variables per point), the suggestion is to monitor the numerical errors, and wherever and whenever the error becomes too large, an additional finer grid is introduced. Similarly, if the error is small enough, the grids are adjusted and possibly removed altogether. Since the error is changing dynamically, this results in a dynamically changing structure of several levels of nested grids.

Of course, the idea of adapting the resolution to the observed numerical error has a long history, and is now commonplace in many areas of numerical computation. For solving initial value problems for ordinary differential equations there are Runge-Kutta methods with adaptive step-size control or the Bulirsch-Stoer algorithm [3]. Adaptive multigrid methods were promoted already in the 1970s by Brandt for

*Electronic address: bruegman@aei-potsdam.mpg.de the solution of elliptic problems in any number of dimensions [4]. For hyperbolic systems the basic reference is the work by Berger and Oliger on adaptive mesh refinement [5]. In the context of general relativity, adaptive mesh refinement has been made famous by Choptuik's pioneering work on the collapse of a spherically symmetric scalar field $[6,7]$. In order to resolve all the details of the Choptuik effect in $1+1$ dimensions, a refinement by a factor on the order of $10^{7}$ over the initial resolution is required. Clearly, if one were to repeat these calculations for more than one spatial variable, the efficiency of adaptive mesh becomes essential.

Perhaps it is appropriate to ask at this point why adaptive mesh, which is such an obvious and simple idea, is not in widespread use in general relativity. There are two main reasons. (As of 1996, we feel it is no longer justified to list limited computer resources as a main reason.)

First, one does have to be able to solve the equations of interest on a uniform grid. This turns out to be a rather hard problem in general relativity, where only a handful of codes in $3+1$ has been developed [8] due to general relativistic problems related to spacetime singularities and the choice of lapse and shift.

Second, programming an adaptive mesh is rather complicated, and it is of a different nature than other programming tasks in numerical relativity because it involves dynamically changing data structures.

In this paper we address both these problems. Based on prior experience with dynamical data structures (in dynamical triangulations for Monte Carlo simulations in fourdimensional Euclidean quantum gravity [16]), it was not too difficult to implement an adaptive mesh code in two and three spatial dimensions. The code was tested as an empty adaptive mesh for a given error function and for the scalar wave equation in flat space.

As a concrete test case in general relativity, we settled on a 3+1 Schwarzschild spacetime in geodesic slicing. Let us emphasize that this is not a showcase for the capabilities of our adaptive mesh code, as only up to three nested grids are involved. But having a general adaptive mesh package available allowed us to automatically use a coarse grid in the outer regions and finer grids near the interior of the black hole. The gain in efficiency in turn allowed us to perform computations on a small workstation that compare well with 
those that the NCSA group performed on various supercomputers [1].

Let us spell out briefly what constitutes the core of our test runs. Given appropriate initial data for the Schwarzschild spacetime at the moment of time symmetry in spatially isotropic, Cartesian coordinates $x, y$, and $z$, the evolution in time $t$ is computed using the standard Arnowitt-DeserMisner (ADM) equations and an explicit finite difference scheme (double leapfrog), where we choose lapse $\alpha \equiv 1$ and shift $\beta^{a} \equiv 0$, which induces geodesic slicing. A point starting at initial Schwarzschild radius $r=2 M$ reaches the singularity at $r=0$ after proper time $\tau=\pi M$, where $M$ is the mass of the black hole. We also evolve the data up to about $\tau=6 \mathrm{M}$ by using the apparent horizon as the inner boundary [17]. The resulting dynamical evolution of, for example, the six metric coefficients can be directly compared to the analytic solution.

The adaptive mesh code can, to a certain extent, be thought of as a black box. The user has to supply just one external input, a routine that evolves data on a uniform grid with a given boundary. While the outer boundary can be incorporated easily into this routine, for the case of an apparent horizon boundary condition it was simpler to customize the adaptive mesh itself, i.e., to incorporate grids with "holes."

The author is aware of two other adaptive mesh refinement packages that are currently under development and that are planned to be applied to $3+1$ numerical relativity: DAGH of the American Grand Challenge Collaboration [18] and a code by Wild [19]. It is interesting to note that the problemindependent design of DAGH does not include grids with holes, but for reasons similar to ours that are specific to general relativity, this feature will be added.

Finally, we want to draw attention to how naturally adaptive mesh fits into general relativity. While the numerical point of view leads us to drive the adaptivity of the adaptive mesh technique by the numerical errors, general relativity gives us a physical reason to split the domain of computation into several grids, namely, simply that one of the main characteristics of general relativity is that spacetime is a manifold, which generically can only be covered by several charts and which can be covered by charts in which the metric is almost flat. This leads us to discuss "numerical relativity on patches" below. In fact, some of the features that make geodesic slicing unattractive for numerical relativity may lose their impact when combined with adaptive mesh. To underscore our point of view we deviate from common terminology and use the term "adaptive mesh" as opposed to the less general "adaptive mesh refinement."

The paper is organized as follows. In Sec. II, we introduce various coordinate systems for the Schwarzschild spacetime and the standard 3+1 decomposition. In Sec. III, we describe our uniform ADM code. In Sec. IV, we discuss some issues related to adaptive mesh in general, while in Sec. V, we present our particular implementation. In Sec. VI, we discuss results obtained for adaptive mesh and Schwarzschild spacetime in geodesic slicing. We conclude with Sec. VII.

\section{SCHWARZSCHILD SPACETIME AND GEODESIC SLICING}

The line element for a single static black hole in Schwarzschild coordinates is given by

$$
d s^{2}=-\left(1-\frac{2 M}{r}\right) d t^{2}+\left(1-\frac{2 M}{r}\right)^{-1} d r^{2}+r^{2} d \Omega^{2},
$$

where $M$ is the mass, $r$ the radius, and $d \Omega^{2}$ the standard line element on the unit two-sphere. We define spatially isotropic coordinates by introducing a new radial coordinate $\bar{r}$, such that

$$
\begin{gathered}
r=\vec{r}\left(1+\frac{M}{2 \bar{r}}\right)^{2}, \\
d s^{2}=-\alpha(\vec{r})^{2} d t^{2}+\psi(\vec{r})^{4}\left(d \vec{r}^{2}+\vec{r}^{2} d \Omega^{2}\right), \\
\alpha(\vec{r})=\left(1-\frac{M}{2 \vec{r}}\right) /\left(1+\frac{M}{2 \bar{r}}\right), \\
\psi(\vec{r})=1+\frac{M}{2 \vec{r}} .
\end{gathered}
$$

This allows us to introduce the Cartesian spatial coordinates that we use in the numerical computations:

$$
d x^{2}+d y^{2}+d z^{2}=d \bar{r}^{2}+\bar{r}^{2} d \Omega^{2}, \quad \bar{r}=\left(x^{2}+y^{2}+z^{2}\right)^{1 / 2} .
$$

The spatially isotropic coordinates possess an isometry at the throat at $\bar{r}=M / 2$ for $\bar{r} \leftrightarrow \vec{r}=M^{2} /(4 \bar{r})$, e.g., $r(\bar{r})=r(\vec{r})$ and $\alpha(\bar{r})=-\alpha(\vec{r})$. The isotropic coordinates for $\bar{r} \in[M / 2, \infty]$ and $\bar{r} \in[M / 2,0]$ cover the same range of the Schwarzschild radius, $r \in[2 M, \infty]$.

In the standard $3+1$ decomposition of the Einstein equations (e.g., [20]), the line element can be written in general as

$$
d s^{2}=-\left(\alpha^{2}-\beta^{a} \beta_{a}\right) d t^{2}+2 \beta_{a} d t d x^{a}+g_{a b} d x^{a} d x^{b},
$$

where $\alpha$ is the lapse function, $\beta^{a}$ the shift vector, and $g_{a b}$ the three-metric. The Einstein equations decompose into the Hamiltonian and diffeomorphism constraint equations, and the evolution equations for the $g_{a b}$ and their canonically conjugate momenta, the extrinsic curvature $K_{a b}$ :

$$
\begin{gathered}
\partial_{t} g_{a b}=-2 \alpha K_{a b}+D_{a} \beta_{b}+D_{b} \beta_{a}, \\
\partial_{t} K_{a b}=-D_{a} D_{b} \alpha+\alpha\left(R_{a b}+K_{a b} K_{c}^{c}-2 K_{a c} K_{b}^{c}\right) \\
+\beta^{c} D_{c} K_{a b}+K_{a c} D_{b} \beta^{c}+K_{c b} D_{a} \beta^{c},
\end{gathered}
$$

where $R_{a b}$ is the three-Ricci tensor, and $D_{a}$ the covariant derivative defined for the three-metric.

The generic evolution problem is, given some initial data for $g_{a b}$ and $K_{a b}$ (solving constraints), a prescription for $\alpha$ and $\beta$, and boundary conditions, construct the spacetime. We make the following choices. For coordinates $t, x, y$, and $z$, we define the initial three-metric at $t=0$ by

$$
{ }^{(3)} d s^{2}=\psi(\bar{r})^{4}\left(d x^{2}+d y^{2}+d z^{2}\right),
$$

where the conformal factor $\psi$ is defined in Eq. (5). The initial data for the extrinsic curvature are determined by making $t=0$ the moment of time symmetry, $K_{a b}=0$. These initial data are a solution to the constraints.

There are several methods to fix the freedom in the definition of the $3+1$ decomposition, and making a good choice 
is essential because otherwise the evolution will break down due to physical or coordinate singularities after a short time. In particular, a lot of work has been carried out on singularity-avoiding slicing conditions (e.g., [1] and references therein). Here we choose geodesic slicing $\alpha \equiv 1$ and $\beta^{a} \equiv 0$, so that points with constant spatial coordinates follow geodesics and $t$ becomes the proper time. The initial data correspond to observers or test particles that are initially at rest and then start falling towards the singularity (no singularity avoidance). For a discussion of problems related to geodesic slicing, see Sec. IV. To test our code we also checked that choosing vanishing shift and the lapse of the quasi-isotropic coordinates, Eq. (4), the configuration does not change [which considering Eq. (9) is a nontrivial numerical problem].

We now have to specify the boundary conditions. As the outer boundary we consider the limit in which $\bar{r} \rightarrow \infty$. In general, there does not exist something like a "purely outgoing wave condition" at finite radius for nonlinear equations such as the Einstein equations, because in general purely outgoing waves are not an exact solution (there always is backscattering). Some approximation is usually the simplest way to proceed, and in our case, similar to [1], it is sufficient to set all fields equal to their initial value at the outer boundary, as long as it is located at $\bar{r}$ sufficiently large. More elaborate procedures are certainly possible, but in conjunction with adaptive mesh not necessary for our problem, since adaptive mesh allows us to go out to sufficiently large values of $\bar{r}$.

We define an inner boundary for intermediate $\bar{r}$ by either using the isometry at the throat, $\bar{r}=M / 2[1]$, or by cutting off the spacetime at the horizon, $r=2 M[17,21]$. In the former case, the isometry defines a simple coordinate transformation from which one can compute the values of the fields for $\bar{r}<M / 2$ once the fields are known for $\bar{r}>M / 2$. Note that $\bar{r}=M / 2$ refers to an unchanging location in our coordinates, but $r=2 M$ defines a curve $\bar{r}=\bar{r}_{\mathrm{ah}}(\tau)$ for the location of the (apparent) horizon. The apparent horizon boundary condition derives from the fact that the horizon is a null surface, so that the exterior is causally disconnected from the interior.

As in [1], to reduce the computational effort by a factor of 8 , most computations are carried out on the octant of positive $x, y, z$ only, and the reflection symmetry of spherical symmetry at the $x=0, y=0$, and $z=0$ planes is used to derive boundary values via a simple coordinate transformation. We did check the code also on the full grid, and it seems quite unlikely that enforcing symmetry only on these planes suffices to ensure spherical symmetry everywhere.

Given the precise evolution problem just stated, what do we know about the resulting spacetime? A convenient feature of geodesic slicing is that the result can be directly compared to the analytic solution. It is somewhat amusing to note that the two previous numerical papers on the topic do not make use of the well-known analytic solution, but in [2] on $1+1$ the validity of the numerical results is established mostly from internal consistency (apart from the crash test), and [1] on $3+1$ check their results against [2]. Of course, in general it is much more useful to be able to check a code without having the analytical solution available, but since it happens to be available in this case, we use it here.

Unit lapse and vanishing shift define Gaussian normal coordinates, which in the context of the Schwarzschild space- time are called Novikov coordinates [22,23]. These are the comoving coordinates in which radially moving freely falling test particles are at rest and the time coordinate measures proper time. Starting from Schwarzschild coordinates, there are several natural coordinate transformations. One can find a transformation to spatially isotropic coordinates, to unit light cones (Kruskal), or to proper time, but of course not simultaneously to proper time and spatial isotropy.

In Schwarzschild coordinates, a radial geodesic starts at $r=0$ and performs a cycloidal motion out to some maximal radius $r_{\max }$ and back to $r=0$. The Schwarzschild geometry in Novikov coordinates is given in terms of a new radial coordinate $R^{*}$ by

$$
\begin{gathered}
R^{*}=\left(\frac{r_{\max }}{2 M}-1\right)^{1 / 2} \\
d s^{2}=-d \tau^{2}+\frac{R^{* 2}+1}{R^{* 2}}\left(\frac{\partial r}{\partial R^{*}}\right)^{2} d R^{* 2}+r^{2} d \Omega^{2},
\end{gathered}
$$

where $r=r\left(\tau, R^{*}\right)$ is implicitly given by the following relation obtained from integrating the geodesic equation:

$$
\begin{aligned}
\frac{\tau}{2 M}= & \pm\left(R^{* 2}+1\right)\left(\frac{r}{2 M}-\frac{[r /(2 M)]^{2}}{R^{* 2}+1}\right)^{1 / 2}+\left(R^{* 2}+1\right)^{3 / 2} \\
& \times \arccos \left[\left(\frac{r /(2 M)}{R^{* 2}+1}\right)^{1 / 2}\right] .
\end{aligned}
$$

To actually compute $r\left(\tau, R^{*}\right)$ we have to invert a relation of the type $y=x+\sin (x)$, which can only be done numerically, but in a very simple manner (e.g., by bisection).

An important property of Gaussian normal coordinates is that the geodesics that define the coordinates remain orthogonal to all constant time hypersurfaces. Therefore, the coordinate transformation between $\bar{r}$ and $R^{*}$ obtained by inserting Eq. (2) into Eq. (11) is time independent. On the other hand, since $r$ is a function of time, the data do not remain isotropic.

To explicitly compute interesting quantities such as the metric coefficients for $M=1, R^{*}>0$, and $\tau>0$, we find it convenient to use the maximal Schwarzschild radial coordinate $r_{\max }$, for which

$$
\begin{gathered}
r_{\max }=2\left(R^{* 2}+1\right)=\frac{(1+2 \bar{r})^{2}}{4 \bar{r}} \\
\tau=r_{\max }\left[\frac{r}{2}\left(1-\frac{r}{r_{\max }}\right)\right]^{1 / 2}+2\left(\frac{r_{\max }}{2}\right)^{3 / 2} \arccos \left[\left(\frac{r}{r_{\max }}\right)^{1 / 2}\right],
\end{gathered}
$$

and, by implicit differentiation,

$$
\frac{\partial r}{\partial r_{\max }}=\frac{3}{2}-\frac{r}{2 r_{\max }}+\frac{3}{2}\left(\frac{r_{\max }}{r}-1\right) \arccos \left[\left(\frac{r}{r_{\max }}\right)^{1 / 2}\right] .
$$

For example, transforming from $R^{*}$ to $\bar{r}$ leads to a simple formula for the radial metric component, 


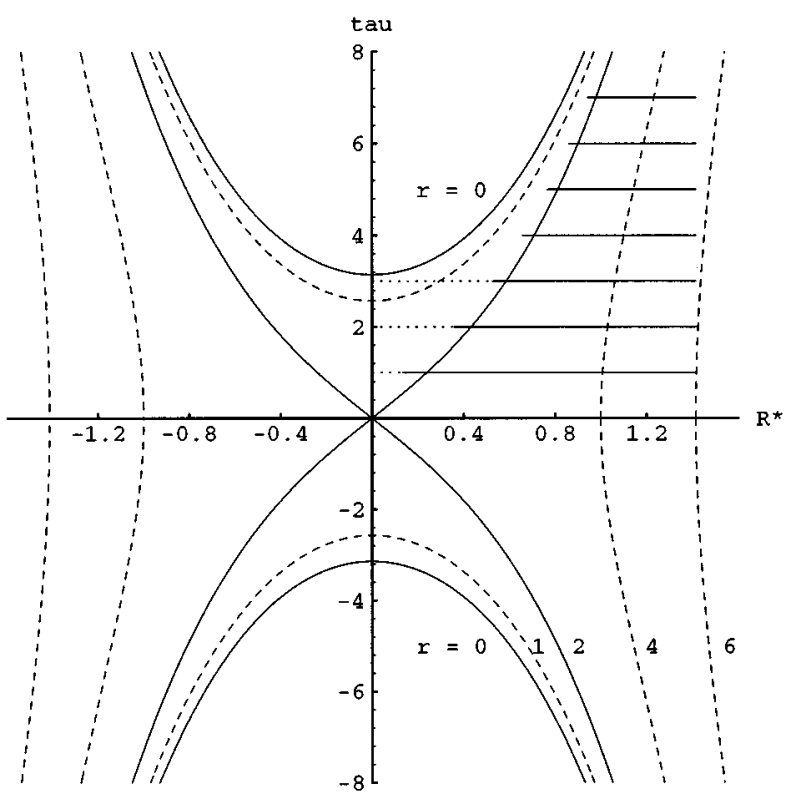

FIG. 1. Novikov coordinates for the Schwarzschild geometry.

$$
g_{\bar{r}} \bar{r}(\tau, \vec{r})=\psi(\vec{r})^{4}\left(\frac{\partial r}{\partial r_{\max }}\right)^{2}\left(r\left(\tau, r_{\max }(\vec{r})\right), r_{\max }(\vec{r})\right)
$$

where as before $g_{\bar{r}} \bar{r}$ depends on time through $r$, which is given implicitly by Eq. (15) as $r\left(\tau, r_{\max }\right)$. Considering that the (time-independent) conformal factor $\psi(\bar{r})=1+M /(2 \bar{r})$ diverges at $\bar{r}=0$, it is natural to compute $g_{a b} / \psi^{4}$ to focus on the dynamical features in the metric rather than on the static $1 / \bar{r}$ singularity, as is done in [1] and as we often do below. Equation (17) justifies this approach.

Figure 1 shows a plot of lines of constant $r$ based on Eq. (13) to depict the Schwarzschild geometry in Novikov coordinates (compare with the qualitative picture in [23]). Note that from Eq. (13) we have for the horizon $\tau / 2 M \approx R^{* 3}$ for large $\tau$, as opposed to Kruskal coordinates in which the horizon is a unit light cone. The horizontal lines show the location of grids with and without apparent horizon boundary conditions.

An initial grid at $\tau=0$ covering $2 M \leqslant r \leqslant r_{0}$ or, equivalently, $0 \leqslant R^{*} \leqslant R^{*}{ }_{0}$ or $M / 2 \leqslant \bar{r} \leqslant \bar{r}_{0}$, moves upwards until the innermost point that started on the horizon reaches the $r=0$ singularity after time

$$
\tau_{\text {crash }}=\pi M
$$

In numerical "crash tests" $[1,2]$ one indeed finds for this scenario that $\tau_{\text {crash }}=(3.1 \pm 0.2) M[1]$, and our $3+1$ code reproduces this result. One can also track how the radial metric component $g_{\bar{r}}(\tau, \bar{r})$ (constructed from $g_{a b}$ ) diverges with time with an exploding peak developing at the throat at $\bar{r}=M / 2[1,2]$. Indeed, from the analytic solution we find for $\tau=3 M$ that at the throat $g_{\bar{r} \bar{r}}=20.486$ compared to 20.2 in $1+1$ and 23.4 in $3+1$ [1].

As a test of our numerical code and in order to present some novel data, we plot $g_{\bar{r}}-\left(\tau, \bar{r}_{\text {ah }}(\tau)\right)$, i.e., how the radial metric component develops with time, in Sec. VI. Having summarized the analytic aspects and some of the numerical history of the Schwarzschild spacetime in Gaussian normal coordinates, we now discuss the actual implementation of our code.

\section{ADM CODE FOR UNIFORM GRIDS}

The evolution equations (8) and (9) for $g_{a b}$ and $K_{a b}$ are rather elegant and concise, but since the indices run from 1 to 3 , writing out each term explicitly leads to a problem of a size that makes the use of computer algebra highly recommended, if not essential for the added flexibility. We wrote a simple MATHEMATICA script that takes Eqs. (8) and (9) directly as input, together with formulas for lapse and shift, and also some control quantities such as the constraints, translates the derivatives into finite differences, and outputs $\mathrm{C}$ code for the basic routine that evolves data on a uniform grid. A typical implementation leads to about 1520 summations, 969 multiplications, and 322 divisions for 18 basic fields.

We choose to perform an unconstrained evolution using explicit finite difference schemes. The schemes tested are Lax-Wendroff, double leapfrog, and Brailovskaya, with and without artificial dissipation (see [2] for a comparison of schemes). As far as data storage is concerned, only the double leapfrog scheme really requires two and not one level of preceding data, that is, the same field at two earlier times. Although this is an additional complication for the adaptive mesh code, we implemented it in order not to introduce a limitation. Most production runs are performed with the double leapfrog scheme. (Compare with [1] where a particular version of staggered leapfrog with extrapolation for the inhomogeneous terms is used.) Second order spatial derivatives are differenced symmetrically with centered differences, which seems to maintain spherical symmetry rather well, although from experience with the Laplace operator and elliptic equations one might expect that some asymmetric differencing is a better choice (e.g., [24]).

In [1], it was observed that for a stable evolution it was crucial to perform differencing of the scaled metric $g_{a b} / \psi^{4}$, which Anninos et al. called conformal differencing. We also had to apply this technique. Since one might argue that the generality of the evolution scheme is compromised by building in knowledge about the initial data (recall that at least $\psi$ does not change with time), let us add a few comments. Clearly, approximating the limiting $1 / \bar{r}$ dependence with finite differences, i.e., essentially with polynomials, is problematic. But as a matter of principle, there always is the issue whether an approximation method works in a given function space. To reduce the problem dependence of conformal differencing, we tested a somewhat more generic method, where a given type of test function, e.g., a rational function, is fitted to the data. The result of the fit is used as a basis for "scaled" differencing. For a perfect fit, one is left with finite differencing a constant. For the problem at hand, however, the simple conformal rescaling was quite sufficient.

As already discussed, at the inner boundary we imposed either the isometry condition or the apparent horizon boundary condition [21]. Not only the field values near the boundary that are needed for the finite difference molecules, but all points in the interior can be obtained by the isometry map from the data that was evolved outside. Since interior points 
are in general mapped to points falling between the outer grid points, a polynomial interpolation is performed, taking due care near the border that no data are accessed before they are available.

For the apparent horizon condition we have postponed the implementation of a general $3+1$ apparent horizon finder, and simply define a surface by the equation $r=2 M$ leading to $\bar{r}=\bar{r}_{\text {ah }}(\tau)$. Following [21] on 1+1, we evolve everywhere outside $\bar{r}_{\text {ah }}(\tau)$ minus some small buffer zone. At various times the innermost points are obtained by second or third order polynomial (or rational function) extrapolation. The basic algorithm can deal with a convex surface which is sufficiently flat on the scale of the grid points. With some finetuning, the inner boundary remains stable with a buffer zone of about 2.8 grid spacings. In [21] a minimal size of 5 and recommended size of 20 grid spacings are reported.

Note that for geodesic slicing all light cones are upright, and so the Courant condition (which requires the physical domain of dependence to be contained in the numerical domain of dependence) reduces to the condition that the angle between the physical characteristics and the $\tau$ coordinate lines is not too large. At large $\bar{r}$, the light cones approach the unit cone $\left(45^{\circ}\right.$ for $\left.c=1\right)$, and it is simple to see that near the horizon the light cones become narrower in the radial direction but wider in the constant radius directions $\left(g_{\tau \tau}=-1\right.$, $g_{\bar{r} \bar{r}}$ increases, and for the polar angle $\theta, g_{\theta \theta}=r^{2}$ decreases).

The numerical domain of dependence is related to the physical one by the factor by which the temporal grid spacing is smaller than the spatial grid spacing. For the finite difference schemes considered, a relative factor of 0.25 was used, although a factor of 0.1 made the evolution slightly more accurate (but slower). We did not encounter the problem that the light cones become too narrow or too wide.

\section{NUMERICAL RELATIVITY ON PATCHES}

Before getting into the details of the adaptive mesh code, we would like to discuss a few issues related to numerical relativity and adaptive mesh in general. As explained in the Introduction, the basic idea is to put points where they are needed for a given accuracy, but in the general relativistic setting a more general viewpoint is possible.

A typical textbook introduction to general relativity may proceed as follows. First one learns that gravitational physics is really about a manifold with a metric. In the neighborhood of any point the manifold looks like $R^{4}$, but in the generic case one needs an atlas of coordinate patches to cover the manifold. Furthermore, there always exist coordinates near a point in which the metric is close to the flat Minkowski metric. To borrow a picture from Einstein's discussion of the principle of general covariance [23], consider the gravitational field of the Earth. Everywhere around the Earth we can construct freely falling frames of reference which approximate Minkowski spacetime, but no single set of coordinates exists in which space time is everywhere flat. So the space time structure with its locally flat patches is a key feature of general relativity, and let us emphasize that, apart from global (topological) issues, it is also a key feature in a practical sense if we look for coordinates in which the metric is locally flat.

Ironically, the next step is to completely ignore or, at

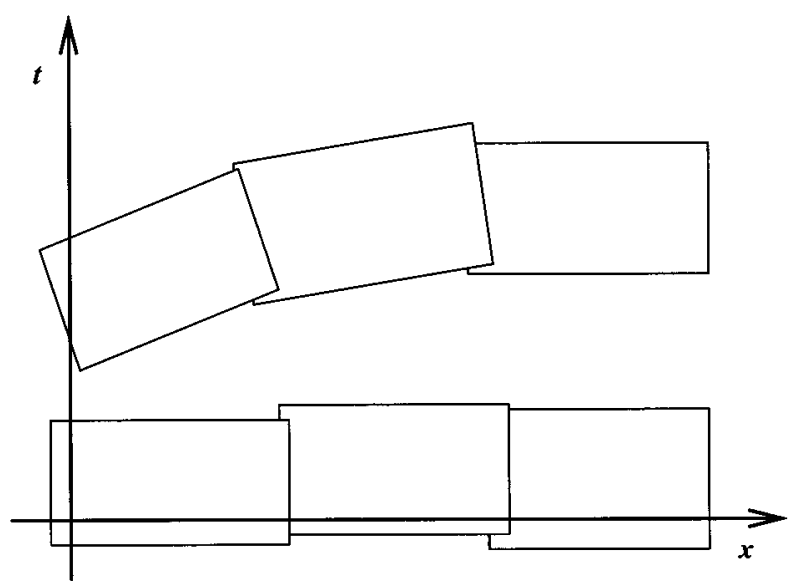

FIG. 2. Schematic example for numerical relativity on patches.

least, circumvent the patch work character of general relativity. One learns a lot about beautiful work where a single or a few coordinate patches are constructed in an ingenious manner to cover all the interesting regions of spacetime [26]. Typically this involves using special symmetries of the model. What is perhaps more relevant for generic $(3+1)$ dimensional numerical relativity without symmetries, for simple initial data the original coordinate system stays good for at least some time close to the initial hypersurface, and so again one might try to make do with one coordinate system rather than changing coordinates.

Numerical relativity has been traditionally built upon one or a few handcrafted grids, mostly fixed for the whole evolution. There are very well-known examples for problems associated with rigid boxes, to name just one, the steep gradients in the metric developing for maximal slicing of the Schwarzschild spacetime [1], with a promising solution being the apparent horizon boundary condition where the grid adapts itself to the apparent horizon and is not strictly fixed.

What we want to suggest is that the adaptive mesh technique encompasses the necessary flexibility to actually implement numerical relativity on patches. Namely, it may be possible to drive the automatic distribution of grids not only by numerical error estimates, but also by some physical measure. For example, such that the new grids correspond to coordinate patches in which the metric is nearly flat or has some other convenient property such as minimal distortion.

Let us emphasize that to us this suggestion appears to be of the type nobody would object to, as long as one can produce a concrete and useful implementation. This is not done here, except perhaps for one aspect discussed below. But we want to develop the idea a little bit further in an illustrative thought experiment for the ADM formalism and for geodesic slicing.

The main idea is displayed in Fig. 2. Suppose we are given initial data that are well represented according to some criterion such as local flatness of the metric. For a brief time, this criterion does not lead us to regrid, and the data evolve in the rectangular spacetime patches that are drawn near the $x$ axis in Fig. 2. Now suppose that Fig. 2 corresponds in a rough sense to a black hole in that in the course of evolution the light cones are tilted inwards toward the $t$ axis and that freely falling observers follow an inward curving path. The flatness criterion could lead at later times to the second row 
of patches, where the initial boxes have been adapted to the inward tilt.

Several comments are in order. Note that the original work by Berger and Oliger [5] already contains the concept of rotating boxes (to track shock fronts), although only in spatial directions. Here the suggestion is to construct boosted data by similar interpolation techniques.

Also note that the data structures are often based on a strict nesting property. Here boxes of equal refinement are allowed to overlap, a feature which one also needs if areas of refinement are to be covered by several small boxes [5].

A technical problem that arises is that in general one has to define the coordinates for each box in an intrinsic manner since there might not exist a single top level parent grid from which all child grids can derive their coordinates. If the spacetime is too warped to be adequately covered by a single grid, several overlapping top level grids are required (compare Fig. 2). A concrete way to implement such parentless grids is to define transition functions between them that must exist since we are dealing with a manifold.

Finally note that no adaptive mesh refinement might be involved at all; i.e., all grids could have the same grid spacing, although one needs a coordinate-independent measure of the grid spacing to make this statement meaningful.

In terms of the standard 3+1 decomposition of Einstein's equations, the above example amounts to a particular choice of both lapse and shift. Introducing a shift vector so that all light cones are upright is the subject of what is called causal differencing [17] or causal reconnection [25]. The difference is that for the particular patches just introduced, the causally correct differencing is discrete on the scale of the grid sizes and not on the scale of the grid spacing. In Sec. III, we discussed that even when the light cones are upright, one still has to adjust lapse and/or temporal and spatial grid spacings. In three spatial dimensions, upright light cones intersect the hypersurfaces in a nonspherical manner, and on the patches one might want to define coordinates such that the cross sections approximate spheres.

To complement this qualitative discussion of numerical relativity on patches, let us conclude the section with a few comments on how the transition from a single, fixed domain of computation to varying patches might be of help for the two main problems that are associated with geodesic slicing. Gaussian normal coordinates have the intrinsic problem that freely falling observers tend to fall into physical singularities and that coordinate singularities develop due to geodesic focusing.

Suppose we had some stable method to stop computing at points where the data become infinite. If all one is given is a fixed finite grid, the grid may have to be unfeasibly large if one wants to cover a given period of time before all points have hit the singularity. But, considering the Schwarzschild spacetime in Fig. 1, even if the outermost points are far enough outside to only move a negligible distance in the time of interest, the innermost points fall in, leading to grid stretching near the horizon. Adaptive mesh is helpful in this regard since it has the built-in capability to introduce new points near the horizon.

For a schematic picture of geodesic focussing, consider Fig. 3. Schwarzschild spacetime is special since all radial geodesics meet at $r=0$. The crucial point is that one has to

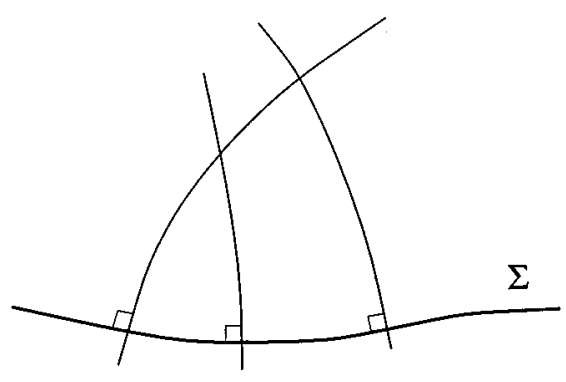

FIG. 3. Geodesic focusing.

determine by some means, e.g., by evaluating curvature scalars, whether one approaches a physical or a coordinate singularity. If there is a physical singularity, then the adaptive mesh can insert finer and finer grids and avoid the singularity, if we decide that this is the feature we want to resolve rather than, for example, to impose an apparent horizon boundary condition (if a horizon covers the singularity). Adaptive mesh, of course, cannot change the underlying physics. If there is a coordinate singularity-and this is the pathological feature of Gaussian normal coordinates we want to address - then quantities such as curvature scalars will appear more and more constant as we approach the intersection of geodesics. The adaptive mesh solution is to regrid, that is, to redistribute points on a coarser grid on a different hypersurface because the finer resolution is not needed since there is no physics to resolve.

We do not seriously want to suggest that geodesic slicing is a universally good choice. For example, having a nonvanishing shift vector might be crucial. But, given a guiding physical principle, adaptive mesh offers the possibility of resolving the problems of geodesic slicing with its built-in capability to add in points where needed when others fell into a physical singularity and to remove points that otherwise would lead to a coordinate singularity. Put the other way around, while on a fixed grid geodesic slicing is certainly problematic, on adaptive meshes these problems are not unavoidable.

The numerical work of this paper can also be considered as a step towards a demonstration that adaptive mesh can fill in points for grids that move and stretch towards a singularity. As the horizon moves outwards, the innermost grid expands to cover the outer regions where points are missing to achieve the given accuracy.

\section{IMPLEMENTING ADAPTIVE MESH REFINEMENT IN TWO AND THREE SPATIAL DIMENSIONS}

All current implementations of adaptive mesh in general relativity derive from Berger and Oliger [5], and are motivated and influenced by Choptuik [6]. We refer to these papers for more technical information, but comment on important features of our code. We should mention that there is at least one nonstandard approach, by Schutz and Wild [19], where the units of refinement are not grids but single points. Our main focus is on $3+1$, but some features are tested in $2+1$ for simplicity. For a visual impression of how the intuitive idea of adaptive mesh translates into various evolving grids that follow some data, see Fig. 4, which is discussed below. 

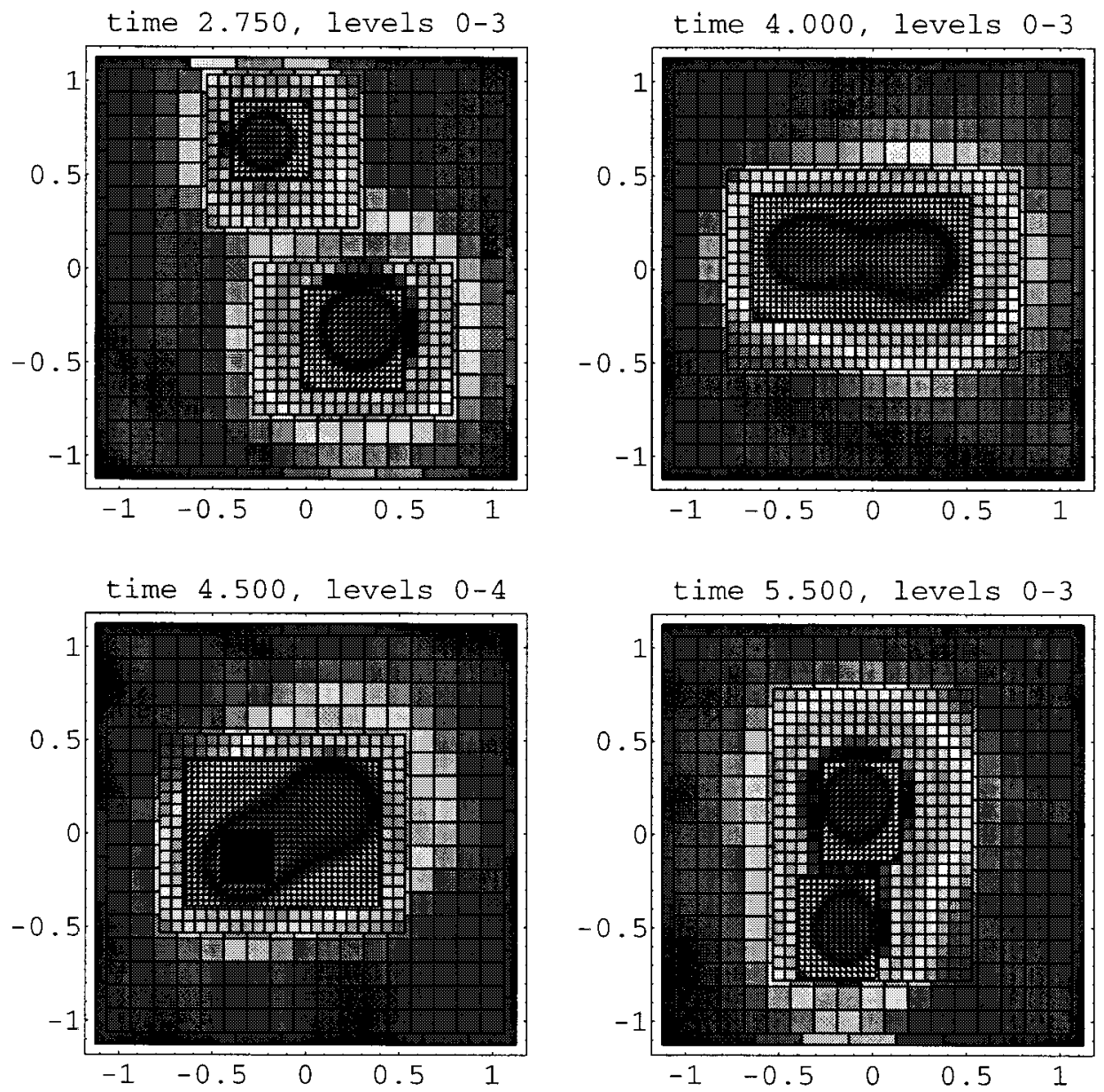

FIG. 4. Empty adaptive mesh displaying various possible regriddings.
The central design issue is how to organize the dynamical data structures. While the basic idea of structuring the grids based on some given error estimate is very simple, it turns out to be a rather complicated matter to have access to all the necessary information at all times. We use linked lists of $c$ structures describing rectangular grids which are referenced by $c$ pointers. This description still leaves a lot of freedom whether one maintains pointers to all or none of the parent grids, the child grids, neighboring grids (we work in three spatial dimensions), equal level grids, and so forth. Depending on the actual physics problem, it becomes a trade-off between the cost to maintain all these pointers versus gain in overall speed. For simplicity, we settled on one choice without worrying about optimization, and for $3+1$ numerical relativity, most time is spent during uniform evolution.

A very helpful idea for testing turned out to be the concept of an empty adaptive mesh. Instead of considering an evolution based on a differential equation, one could consider some fake evolution, for which one also specifies a fake error function. But all that the adaptive mesh is adapting to is the error function, and so we are considering empty adaptive meshes which track the evolution of some predetermined error function without reference to any data.

Figure 4 shows two regions of error circling each other in $2+1$ dimensions. This models the situation of a neutron star binary, for which we might also expect the error to be large where the density is high (although this is not necessarily the case). The color coding is normalized separately for each grid to set off the subgrids. The fine grids follow the peaks, boxes of equal refinement merge and split, and finer boxes are inserted and removed.

One part of the adaptive mesh code is to find appropriate boxes around volumes where the error is beyond a certain threshold or, equivalently, to find the bounding box for flagged points among unflagged points. To find rectangular bounding boxes, we start with a seed and let each of its faces move outwards in turn as long as there are flagged points on it, and since the volume grows, we have to repeatedly consider each surface. The optimal performance is obtained for sets of flagged points which form solid boxes, since starting with any seed, it is a linear process to walk out to the surface, and in order to decide that the final surface does not contain flagged points, $O\left(N^{2}\right)$ operations are required for a box of volume $N^{3}$. Actually, even if we had to look at each single point inside the final box a few times, the time spent on finding boxes would be negligible compared to on the order of $1000 N^{3}$ floating point operations carried out per point during evolution. Note that this algorithm will group disconnected regions when appropriate (e.g., nonconvex regions whose bounding boxes overlap), which is a big advantage over certain flood-fill algorithms.

Note also that putting an upper limit on the volume to which a seed may grow offers a simple way to break up large regions into several small boxes. We have not implemented this yet, but this certainly is a good way to improve efficiency once storage for on the order of 100 reasonably sized boxes is available (as opposed to a current limit of about 5 in $3+1)$. For example, the black hole space time we consider 


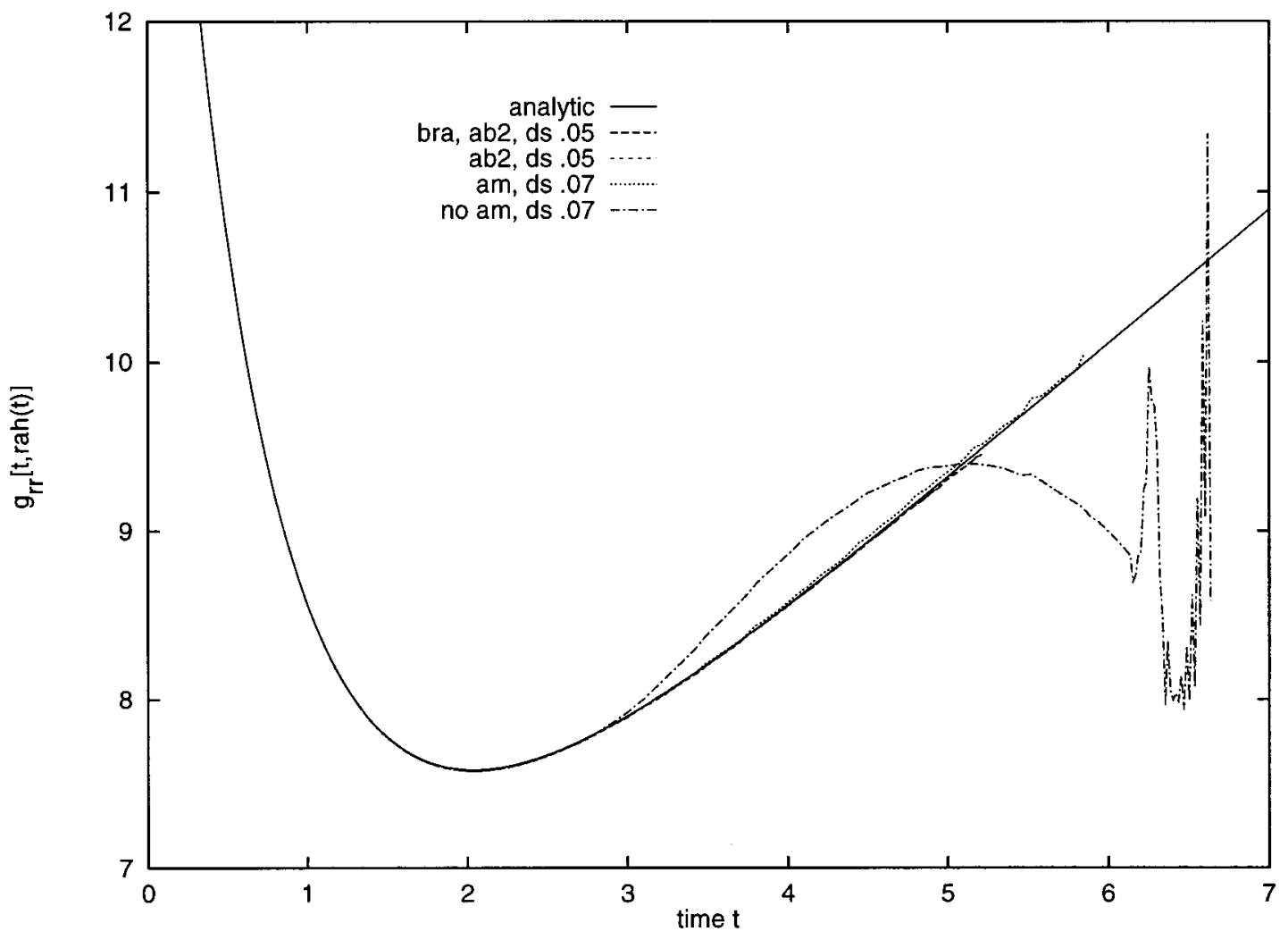

FIG. 5. The metric component $g_{\bar{r} \bar{r}}$ at the horizon.

poses the problem to cover a spherical shell, the region near the horizon, which contains far fewer points than the bounding box. Referring to Sec. IV, spatially nonuniform refinement can be useful, but since the refinement factor is constant in each grid, one needs a larger number of boxes to adequately break up large nonuniform regions.

One aspect of adaptive mesh that is not testable in empty adaptive mesh by its very definition is how the error estimates are obtained. As usual, we compute the Richardson truncation error, which involves comparing data from the evolution on coarse and fine grids.

Another very important issue not adressed with empty adaptive mesh is the question of how to obtain the boundary data for the interior subgrids. We refine the grid spacing for both space and time by the same factor (any integer larger than 1), and so there are time steps for which a grid is not covered by a coarser grid at equal time from which the boundary could be interpolated. But evolving the coarser grids first, any subgrid is always sandwiched between two coarser grids in time. The coarsest grid is only allowed to have outer boundaries, which have to be treated by different means anyway. In our examples it has worked well to derive the boundary for the finer grids by polynomial interpolation of order no higher than 3 from the two coarser grids. We tested the interpolation first for a scalar field in $2+1$ and $3+1$ dimensions (planar and spherical waves) before proceeding to the black hole case.

It is not clear whether the interior boundaries introduced by the adaptive mesh can be treated completely independently of the evolution scheme as we do it here with the above interpolation scheme. As already mentioned in the Introduction, we found it useful to open up the black box con- cept for adaptive mesh somewhat by incorporating the apparent horizon boundary condition as boxes with holes, even though the apparent horizon is an outer boundary of the domain of computation. Also recall that we have experimented only with explicit difference schemes for an unconstrained evolution. Whenever a nonlocal operation has to be performed, for example, in an implicit difference scheme or when solving an elliptic boundary value problem, it is still possible to evolve the coarse grid first for the region where the coarse data are valid. But the nonlocality might introduce a new source of noise into the system.

Regridding noise is the one additional numerical problem introduced by adaptive mesh. Every time the grids change, there will be an unavoidable numerical error due to interpolation and injection of data. In our examples, a sufficiently fine grid spacing kept the regridding noise at small enough levels. Artificial dissipation reduces the noise, but was not essential.

\section{ADAPTIVE MESH AND GEODESICALLY SLICED SCHWARZSCHILD SPACETIME}

In this section we present results of our $3+1$ ADM adaptive mesh code for the Schwarzschild space time in geodesic slicing. In Fig. 5 we plot the unscaled metric component $g_{\bar{r} \bar{r}}$ at time $\tau$ on the horizon at radius $\bar{r}_{\mathrm{ah}}(\tau)$. The data is taken on the diagonal of the first octant. We set $M=1$ in this section. Five different data sets are plotted. The solid smooth line is the analytical result. At time $0, \bar{r}_{\mathrm{ah}}=\frac{1}{2}$ and $g_{\bar{r} \bar{r}}=16$ [compare Eqs. (5) and (10)]. Initially, there is a drop in $g_{\bar{r} \bar{r}}$ as the horizon moves away from the $1 / \bar{r}$ singularity in the conformal factor, while at late times, $\bar{r}_{\mathrm{ah}} \propto \tau^{2 / 3}$ and also 

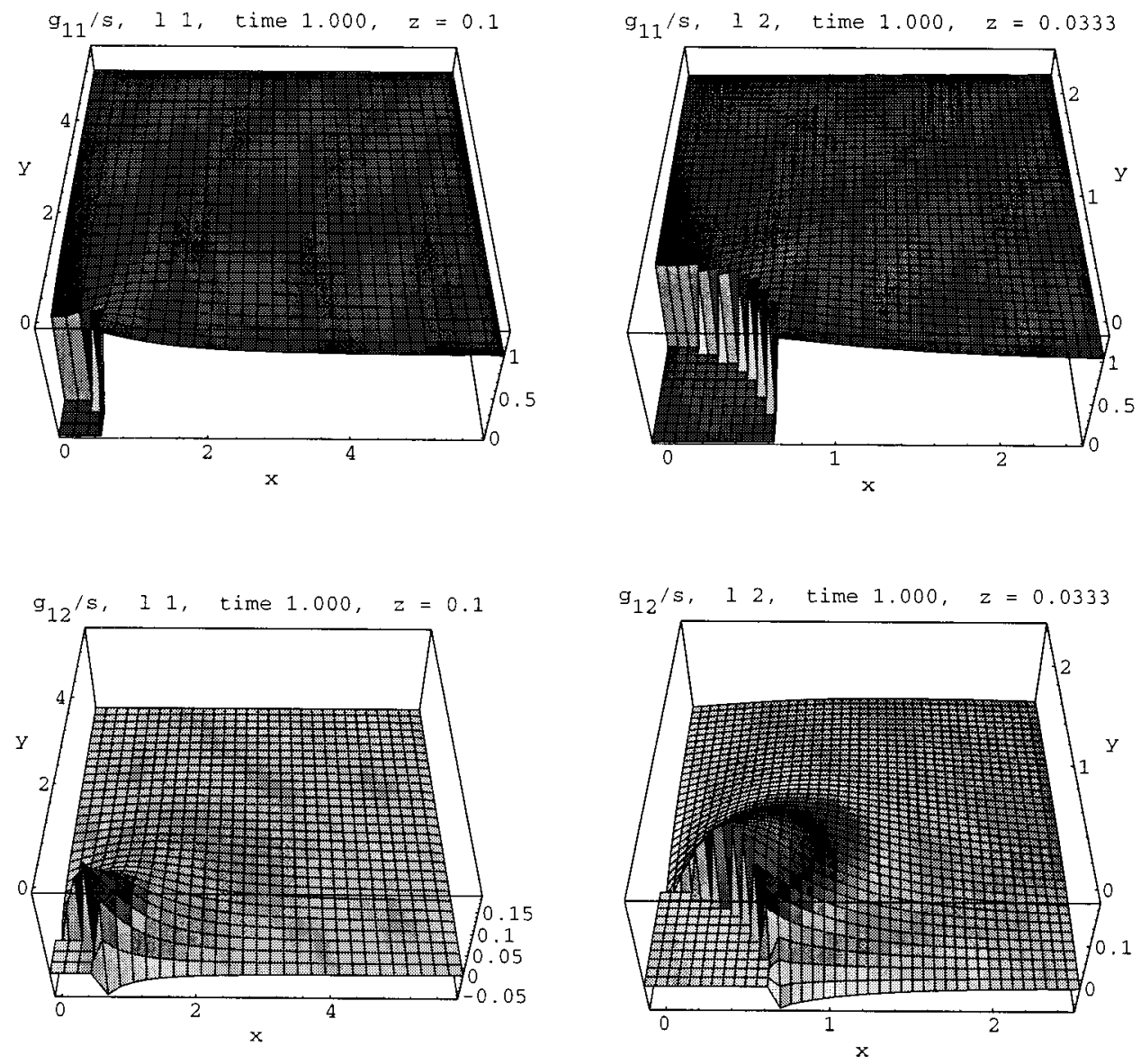

FIG. 6. Metric components at levels 1 and 2 at time $\tau=1.0$.
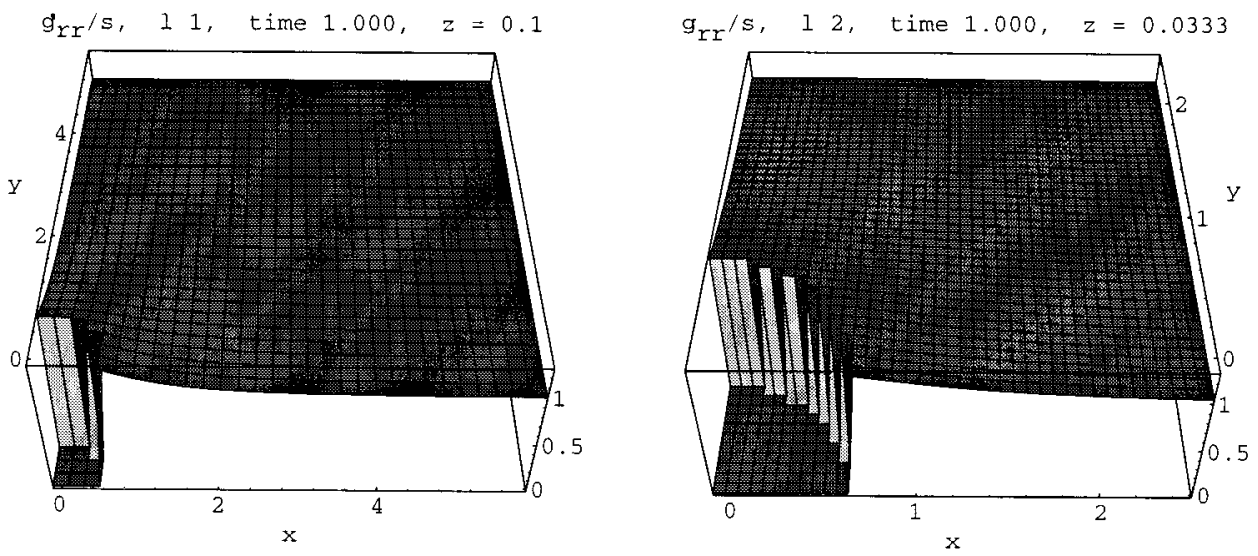

$g_{\bar{r} \bar{r}} \propto \tau^{2 / 3}$, so that $g_{\bar{r} \bar{r}} \propto \bar{r}_{\mathrm{ah}}$. These estimates are valid for $\bar{r}_{\text {ah }}$ large enough so that $\psi\left(\bar{r}_{\mathrm{ah}}\right) \approx 1$, but note that at the right edge of Fig. $5, \bar{r}_{\mathrm{ah}}(7)=2.8$ and $\psi\left[\bar{r}_{\mathrm{ah}}(7)\right]^{4}=1.9$. The horizon moves out, but so does the $r=0$ singularity, and it just so happens that the horizon marks a value on the flank of the $r=0$ singularity in the radial metric that moves to infinity increasing linearly with the radial coordinate of the metric.

The main result of this paper is the line slightly above the analytic curve. It is obtained on the finest level of a three level adaptive mesh with grid spacings $0.07,0.21,0.63$ (refinement factor 3 ). The computations are performed in the first octant with double leapfrog and conformal differencing, and without artificial dissipation. At the inner boundary an apparent horizon boundary condition is used with three buffer points, and the coarse grids reach far enough to hold the data constant at the outer boundary.

In Figs. 6 and 7 we plot for the same run two-dimensional cuts of $g_{\bar{r}} \bar{r}, g_{x x}$, and $g_{x y}$ scaled by $\psi^{4}$ at $\tau=1.0$ and $\tau=5.5$, respectively. The two finest levels are shown. Level 0 does not extent further than level 1 and is just maintained for the truncation error estimate. The data inside the horizon minus buffer are arbitrarily set to zero since no evolution is computed there. Note that at $\tau=5.5$ the horizon has almost reached the border of the level-2 grid. A small inaccuracy is visible at the boundary of the level- 1 grid at $\tau=5.5$ due to the constant outer boundary condition. There is a corresponding deviation from zero in the Hamiltonian constraint, which propagates through the whole domain of integration 

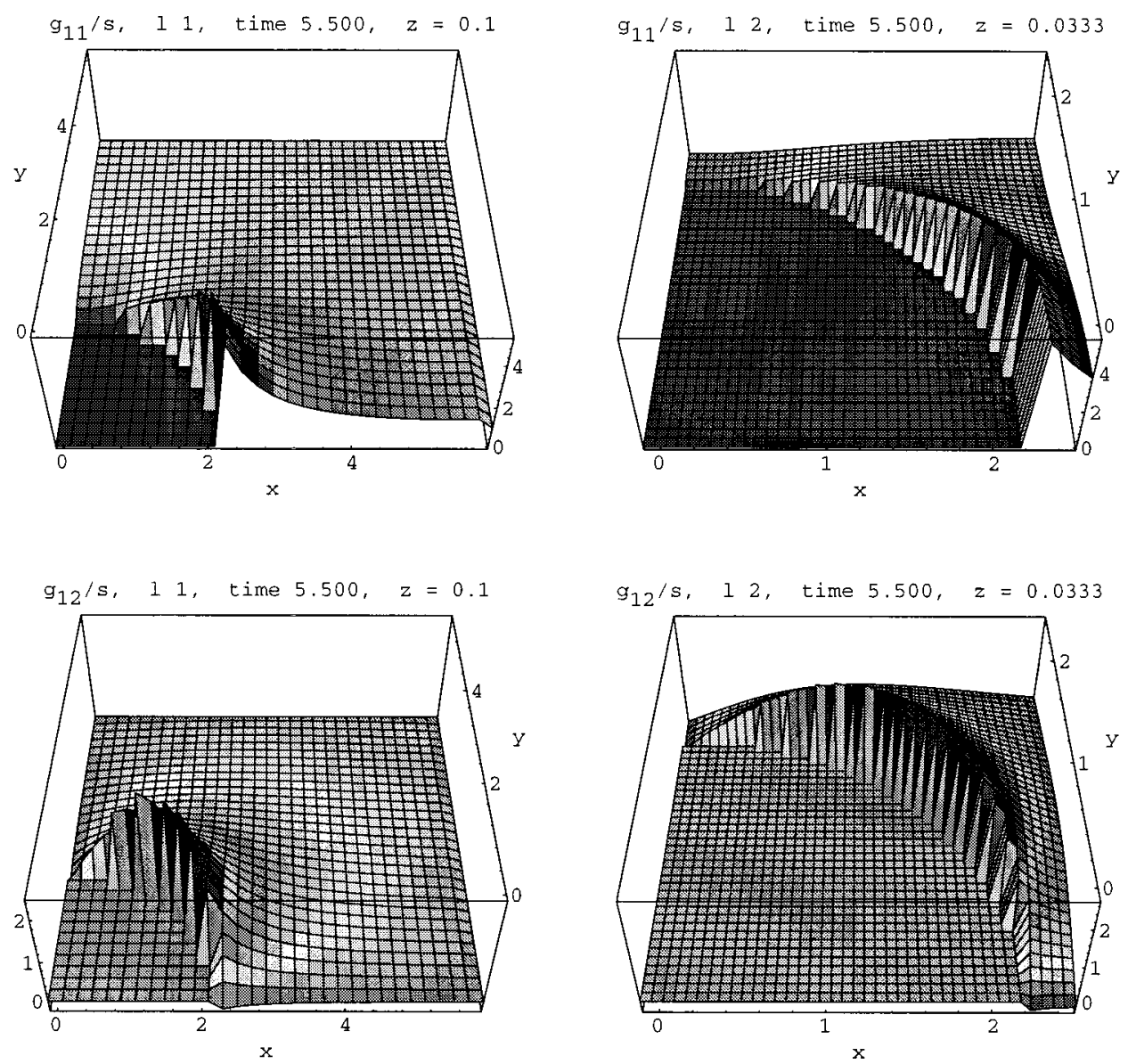

FIG. 7. Metric components at levels 1 and 2 at time $\tau=5.5$.
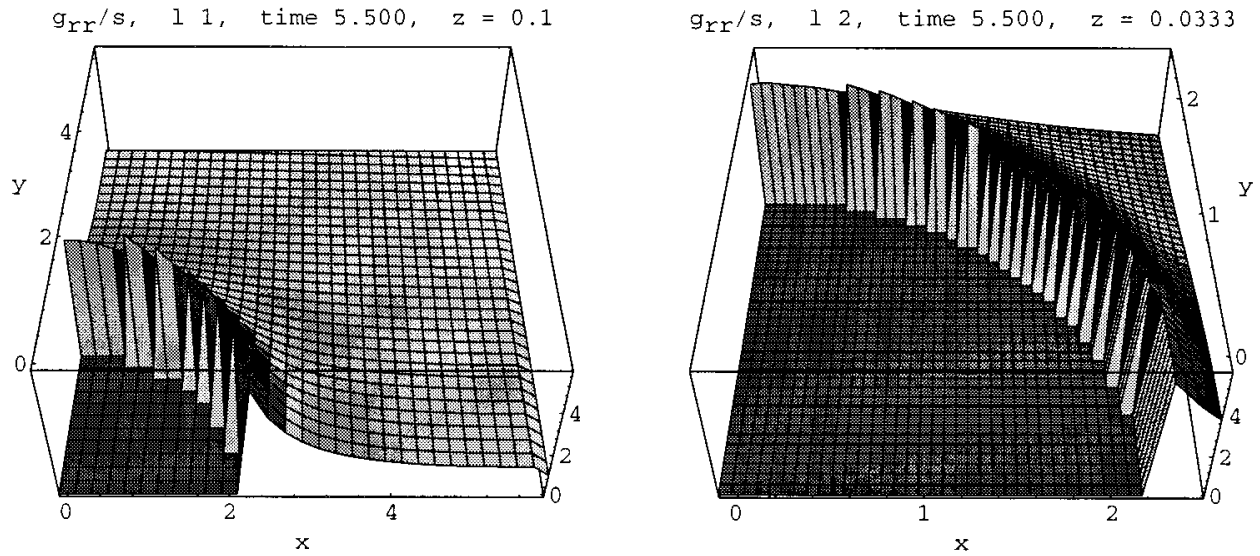

but remains small. Experience shows that it is far from simple to obtain a stable evolution for the gradients in level-1 at $\tau=5.5$. But this is one of the problems that is reduced by adaptive mesh, since as usual the data from the level-2 grid have been injected.

The curve that deviates wildly from the analytic solution at late times in Fig. 5 belongs to a single level run under identical conditions as defined above except that no coarser grids are introduced. There are two further runs plotted in Fig. 5, which fall just slightly below the analytic curve. They correspond to uniform grids with spacing 0.05 and analytic data at the outer boundary. One of the runs was performed with the Brailovskaya scheme, which in our implementation is twice as slow as double leapfrog and does not improve accuracy as opposed to [2].

The key limiting factor of all these runs is computer memory. Typical runs involve one or two boxes with about $40^{3}$ points on a 24 Mflop machine (linpack.c) with $80 \mathrm{MB}$ RAM taking $10 \mathrm{~h}$ (compared to gigaflops, gigabytes, and about the same time at NCSA [1]). Having 40 points in any one direction is ridiculously little compared to what is available for lower dimensional problems. In conjunction with adaptive mesh it is clearly much more efficient to have 2 boxes of size $40^{3}$ rather than one box of $50^{3}$, for which the total number of points is about the same. In [1], for geodesic slicing a grid of size $128^{3}$ is used with grid spacing 0.05 to 
cover about the same range of $x=0-6$ as in Figs. 6 and 7 . Up to $200^{3}$ was manageble in [1]. We find it surprising how well one can do with so few points per direction and a grid spacing which by no means is "much smaller" than 1 .

The apparent horizon boundary condition is working well. Even with only about three points as a buffer zone, data directly at the horizon are not significantly affected on this scale, which is apparent in Fig. 5, and which we also checked by comparing with runs for analytic inner boundary.

There are several reasons why the runs in Fig. 5 cannot be continued to later times, all of them related to size limitations. The truncation errors that drive the adaptivity are spherically distributed, and given the current resolution we do not attempt to cover spheres by several grids; so all grids are concentric about $\bar{r}=0$. Hence, given some maximal volume such as $40^{3}$, the grid spacing determines the position of the outer boundary. Referring to Fig. 1 for Schwarzschild in Novikov coordinates, it is clear that at late times there is no room for the three-point buffer necessary for the apparent horizon condition. Even before that, the steep increase in the metric coefficients makes the evolution unstable. So in these coordinates at this resolution we are squeezed out at around $\tau=6$.

In [1], evolution times of around $t=15-50$ have been obtained, which is the best one has achieved in $3+1$ dimensions, but for different coordinates (various implementations of maximal and algebraic slicing, horizon locking shift). For the accurate extraction of gravitational waves, on the order $t=1000$ would be nice. Geodesic slicing is not well suited for a code that is supposed to run forever, because the horizon keeps moving outward and the radial metric coefficients increase. For the same reason, geodesic slicing makes for an interesting test case apart from the crash test, because one can work on some aspects of moving horizons.

The one scheme without built-in time limitations is based on horizon locking shift conditions [21]. One starts with dy- namically evolving data, but manages to find coordinates in which the metric becomes static, which unsurprisingly is possible for the Schwarzschild spacetime. To find the final static black hole is just what one needs for many problems; on the other hand, our maximal proper time of $\tau=6$ is not too bad for a genuinely dynamical slicing.

\section{CONCLUSIONS AND OUTLOOK}

The numerical results collected with our new $(3+1)$ dimensional adaptive mesh code in the case of Schwarzschild spacetime in geodesic slicing are in good agreement with the analytical solution. Tests of the adaptive mesh rely mostly on no physics and flat space scenarios, but for the black hole case the added efficiency of the adaptive mesh was crucial for performing the computations on a small workstation. The evolution reproduces the crash time of $\pi M$, and can also be carried out to about $6 M$ with the help of an apparent horizon boundary condition. We argued that numerical relativity on patches is a natural idea for adaptive mesh in general relativiy.

Apart from obvious extensions of this work to larger machines, let us mention three directions for future work. The adaptive mesh can be generalized to cover some aspects of numerical relativity on patches, e.g., to overlapping boxes without parents. Having the ADM compiler available, one can experiment with the various hyperbolic formulations that have become available recently (see [27] for a review). Finally, as a simple example for nonvacuum general relativity, one can study the collapse of a scalar field in $3+1$ to find out whether the Choptuik effect exists for nonspherical configurations of the scalar field.

\section{ACKNOWLEDGMENTS}

It is a pleasure to thank G. Allen, B. Schmidt, and B. Schutz for helpful discussions.
[1] P. Anninos, K. Camarda, J. Massó, E. Seidel, W. Suen, and J. Towns, Phys. Rev. D 52, 2059 (1995).

[2] D. Bernstein, D. Hobill, and L. Smarr, in Frontiers in Numerical Relativity, edited by C. Evans, L. Finn, and D. Hobill (Cambridge University Press, Cambridge, England, 1989).

[3] W. Press, S. Teukolsky, W. Vetterling, and B. Flannery, Numerical Recipes in $C$ (Cambridge University Press, Cambridge, England, 1992).

[4] A. Brandt, in Multigrid Methods, edited by W. Hackbusch and U. Trottenberg, Springer Lecture Notes in Mathematics No. 960 (Springer-Verlag, Berlin, 1982).

[5] M. Berger and J. Oliger, J. Comput. Phys. 53, 484 (1984).

[6] M. Choptuik, in Approaches to Numerical Relativity, edited by R. d'Inverno (Cambridge University Press, Cambridge, England, 1992).

[7] M. Choptuik, Phys. Rev. Lett. 70, 9 (1993).

[8] If one asks of a "true", $(3+1)$-dimensional code to be able to compute the evolution for at least vacuum general relativity in three independent spatial variables, but allows that only certain types of initial data can be evolved, possibly only for a short but nontrivial time interval, then there are the following implementations. We already mentioned Anninos et al. [1]. The NCSA group has also implemented both a standard and a flux conservative scheme for weak gravitational waves; for first results see [9] and, also, a technical paper is in preparation. Bona and Massó report on a flux conservative code for inhomogeneous pancake collapse already in [10]. Allen et al. are developing a code for a moving black hole [11]. While not strictly in the above category, we have to mention the early work on binary neutron stars in $3+1$ dimensions by Nakamura et al. [12]. Shibata and Nakamura have implemented a fully relativistic 3D code for gravitational waves [13]. An important role will be played by apparent horizon finders in $3+1$; see Anninos et al. [14] and Baumgarte et al. [15].

[9] P. Anninos, J. Massó, E. Seidel, W. Suen, and M. Tobias, Phys. Rev. D (to be published).

[10] C. Bona and J. Massó, in Approaches to Numerical Relativity, edited by R. d'Inverno (Cambridge University Press, Cambridge, 1992).

[11] G. Allen, M. Alcubierre, S. Farrar, B. Schutz and L. Wild, in General Relativity, Proceedings of 7th Marcel Grossman 
Meeting, Stanford, California, 1994, edited by R. Ruffini and M. Keiser (World Scientific, Singapore, 1995).

[12] T. Nakamura, K. Oohara, and Y. Kojima, Prog. Theor. Phys. Suppl. 90, 1 (1987).

[13] M. Shibata and T. Nakamura, Phys. Rev. D 52, 5428 (1995).

[14] P. Anninos, D. Bernstein, S. Brandt, J. Libson, J. Massó, E. Seidel, L. Smarr, W. Suen, and P. Walker, Phys. Rev. Lett. 74, 630 (1995).

[15] T. Baumgarte, G. Cook, M. Scheel, S. Shapiro, and S. Teukolsky, Phys. Rev. D 54, 4849 (1996).

[16] See, for example, B. Brügmann and E. Marinari, Phys. Rev. Lett. 70, 1908 (1993); J. Math. Phys. (N.Y.) 36, 6340 (1995); B. Brügmann, Phys. Rev. D 47, 3330 (1993).

[17] E. Seidel and M. Suen, Phys. Rev. Lett. 69, 1845 (1992).

[18] E. Seidel (private communication).

[19] B. Schutz (private communication); see L. Wild, Ph.D. thesis, Cardiff University, Wales, in preparation, 1996.

[20] J. York, in Sources of Gravitational Radiation, edited by L.
Smarr (Cambridge University Press, Cambridge, England, 1979).

[21] P. Anninos, G. Daues, J. Masso, E. Seidel, and W. Suen, Phys. Rev. D 51, 5562 (1995).

[22] I. Novikov, Ph.D. thesis, Shternberg Astronomical Institute, Moscow, 1963

[23] C. Misner, K. Thorne, and J. Wheeler, Gravitation (W. H. Freeman and Company, San Francisco, 1973), p. 826.

[24] A. Mitchell and D. Griffiths, The Finite Difference Method in Partial Differential Equations (John Wiley \& Sons, New York 1980).

[25] M. Alcubierre and B. Schutz, in Approaches to Numerical Relativity, edited by R. d'Inverno (Cambridge University Press, Cambridge, 1992); J. Comput. Phys. 112, 44 (1994).

[26] I thank T. Newman for pointing out that even for the Schwarzschild spacetime there exists a single complete chart, e.g., T. Newman and D. Pajerski, J. Math. Phys. (N.Y.) 12, 1929 (1971).

[27] H. Friedrich, “'Hyperbolic reductions for Einstein's field equations,', Report No. AEI001, Potsdam, 1996 (unpublished). 